\title{
Correspondence
}

\section{Direct antiviral agents for treatment-naïve patients with genotype 1 hepatitis C: A statisti- cal model for comparing outcomes between real world and clinical trials}

\section{Dear Editor,}

In pharmacotherapy, evidence-based analyses frequently aim to compare the outcomes observed in the real world (i.e., observational studies) with the effectiveness suggested by clinical trials (e.g., phase III experimental studies) [1]. The typical analytical strategy is retrospective: when the final results are available for both the experimental and the observational studies, a series of statistical tests are undertaken to determine if the latter agree with the effectiveness reported in the former $[3,4]$.

In the treatment of hepatitis $C$ (particularly, genotype 1 infection), clinical trials [2] as well as observational studies in the real world on the newest direct antiviral agents (DAAs), have been performed. Some observational experiences have already been published as clinical reports or in the framework of costeffectiveness studies [3].

The analysis presented herein is focused on the treatment of hepatitis $\mathrm{C}$ virus (HCV) genotype 1 , treatment-naïve patients with the combination sofosbuvir + ledipasvir [2-4]. In particular, we applied an original Bayesian model [5] in which the comparison between observational and experimental outcomes is iteratively repeated over time as more and more observational patients are enrolled ("continuous monitoring" [5]). The objective of this comparison is to determine, from a statistical viewpoint, if the observational results (monitored at different stages of patients' enrolment) agree with those previously reported in experimental trials.

In comparison with traditional frequentist methods, the Bayesian approach has an advantage because its statistical model incorporates information from previous populations. In this way, a combined parameter estimation is performed that uses not only the data from the patients under study, but also information from previous populations. This generally leads to more conservative results than those obtained by the frequentist approach.

In the treatment of genotype 1 infection with sofosbuvir + ledipasvir, the expected success rate suggested by clinical trials in treatment-naïve patients (sustained virological response 12 weeks after treatment completion) is $96-97 \%$ [3]. In our primary analysis, we employed the value of $96.5 \%$ and, according to the Bayesian model [5], we set the inferiority boundary at 76.5\% therefore assuming inferiority for success rates less than $76.5 \%$ (cut-off to
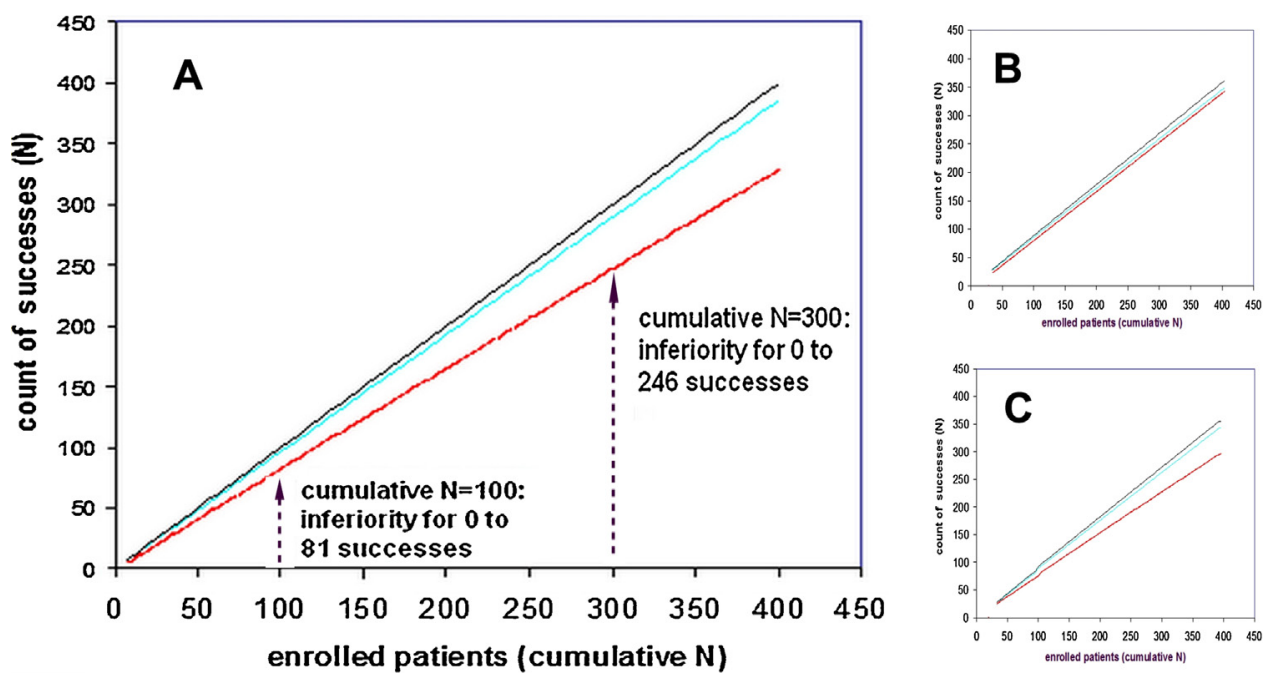

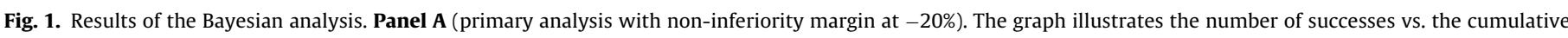

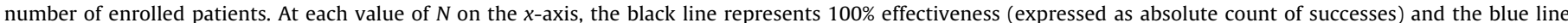

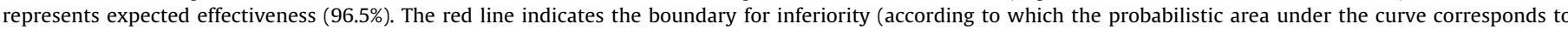

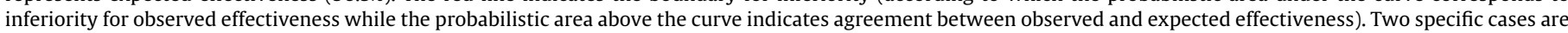

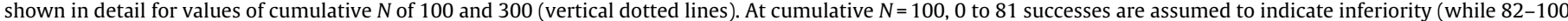

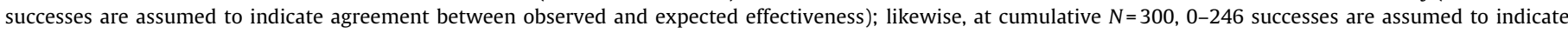

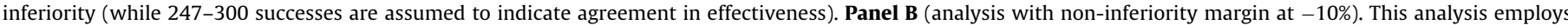

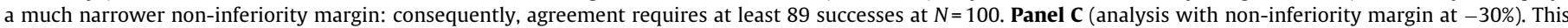
analysis is employs a more "flexible" non-inferiority margin: agreement is satisfied if only 75 successes are achieved at $N=100$. 
stop for inferiority i.e., fail to reject $\mathrm{H} 0$ if $\operatorname{Pr}(\mathrm{H} 1 \mid$ Data $)<0.05$; cut-off to stop for superiority i.e., reject HO if $\operatorname{Pr}(\mathrm{H} 1 \mid$ Data $)>0.95)$. Continuous monitoring of enrolled patients was for every 4 new patients enrolled; in particular, for each number of enrolled patients, two ranges of absolute success counts were estimated indicating, respectively, inferiority of real patients in comparison with expected effectiveness or agreement between observational and experimental outcomes.

The results of our primary analysis (Fig. 1, Panel A; noninferiority limit set at $-20 \%$ ) show how our the Bayesian model manages this continuous monitoring of outcomes by distinguishing 'agreement with expected results' from 'inferiority'. Clearly, this distinction is extremely helpful in practical terms, mainly because situations of poor effectiveness, when present, can be recognised without delay with methodological rigour. One critical issue in this analysis is the choice of the cut-off in the success rate below which inferiority is assumed (76.5\% in our analysis). We set this absolute reduction value at $-20 \%$ mainly because observational evidence always tends to be less than in clinical trials. Anyhow, two secondary analyses are presented in Fig. 1 (Panels B and C), where the non-inferiority limits were set, respectively, at $-10 \%$ and $-30 \%$. Since this is the most critical parameter of the model, the results of the analysis change in response to changes of this boundary. The detailed values of the boundary for any value of cumulative $N$ are reported in Supplementary Table S1.

A number of clinical factors (related to patients' characteristics, such as non-adherence, drug interactions, co-morbid events and toxicity) can contribute to reduce the effectiveness in the real world, and this is likely to occur also in hepatitis C. Furthermore, hepatitis $C$ can have a further peculiarity related to the high cost of treatment: when DAAs are made available to patients' free of charge through the national health system, one explanation for ineffectiveness - that goes beyond the traditional medical scenario - is that a patient can intentionally omit to take the drugs to resell them on the black market.

This study had the purpose of describing the model along with an example of application. Further analyses will be needed to study other cohorts identified on the basis of other criteria including type of treatment, naïve vs. pre-treated patients, presence of cirrhosis, degree of fibrosis, or intolerance to interferon [3].

\section{Conflict of interest}

None declared.

\section{Appendix A. Supplementary data}

Supplementary material related to this article can be found, in the online version, at http://dx.doi.org/10.1016/j.dld.2015.06.012

\section{References}

[1] Rothwell PM. Commentary: External validity of results of randomized trials: disentangling a complex concept. International Journal of Epidemiology 2010;39:94-6.

[2] Alqahtani SA, Afdhal N, Zeuzem S, et al. Safety and tolerability of ledipasvir/sofosbuvir with and without ribavirin in patients with chronic $\mathrm{HCV}$ genotype 1 infection: analysis of phase 3 ION trials. Hepatology 2015;62:25-30.

[3] Chhatwal J, Kanwal F, Roberts MS, et al. Cost-effectiveness and budget impact of hepatitis C virus treatment with sofosbuvir and ledipasvir in the United States. Annals of Internal Medicine 2015:162:397-406.

[4] European. Association for the study of the liver EASL recommendations on treat ment of hepatitis C 2015. Journal of Hepatology 2015;63:199-236.

[5] Johnson VE, Cook JD. Bayesian design of single-arm phase II clinical trials with continuous monitoring. Clinical Trials 2009;6:217-26.

Andrea Messori

HTA Unit, Regional Health Service, ESTAR-Tuscany Region, Florence, Italy
Maurizia R. Brunetto

Hepatology Unit, Reference Center of the Tuscany Region for Chronic Liver Disease and Cancer, University Hospital of Pisa, Pisa, Italy

Andrea De Luca ${ }^{\mathrm{a}, \mathrm{b}}$

a Infectious Diseases Unit, Azienda Ospedaliera Universitaria Senese, Siena, Italy

${ }^{\mathrm{b}}$ Department of Biotechnology, University of Siena, Siena, Italy

Anna Linda Zignego Department of Internal Medicine, Center for Systemic Manifestations of Hepatitis Viruses, University of Florence, Florence, Italy

* Corresponding author at: HTA Unit, Regional Health System of Tuscany, Via San Salvi 12, 50100 Firenze, Italy. Tel.: +39338 9513583; fax: +390574701319 .

E-mail address: andrea.mesori.it@gmail.com

(A. Messori)

http://dx.doi.org/10.1016/j.dld.2015.06.012

\section{High intensity focused ultrasound for the treat- ment of advanced liver cancer}

\section{Dear Editor,}

A 61-year-old female patient with Child C liver cirrhosis due to chronic alcoholic disease was diagnosed with hepatocellular carcinoma (HCC) by imaging findings. The lesion was subcapsular and subdiaphragmatic $(3.5 \mathrm{~cm})$ (Fig. 1).

Surgical resection or liver transplantation could not be considered as therapeutic options because of the patient's decreased general condition and ongoing alcohol abuse. Treatment with sorafenib was contraindicated due to Child C cirrhosis (MELD score 25). Furthermore, minimally-invasive local ablative techniques could not be safely performed because of tumour location.

After tumour board review, treatment approach with highintensity focused ultrasound (HIFU) was approved. Although transarterial chemoembolization of HCCs before HIFU may enhance tumour susceptibility for therapeutic ultrasound [1], this was not feasible due to impaired liver function.

HIFU-ablation was performed using an ultrasound-guided device (Supplementary Figs. S-1 and S-2). No periprocedural complications occurred. Imaging 3 days and 12 weeks after HIFU showed a completely devascularized lesion without evidence for residual tumour (Fig. 1). Clinical course was uneventful.

In advanced HCC stages, minimally-invasive ablative techniques often allow a satisfactory local tumour control. However, these methods are based on the percutaneous insertion of needle-like devices. Moreover, size of ablation zone and boundary to healthy tissue is not precisely predictable. Their use is therefore limited to selected locations due to risk of vascular, biliary, cardiac or diaphragmatic injury.

HIFU may overcome these limitations. It is a non-invasive method that causes coagulation necrosis in the targeted lesion by focused ultrasound energy.

HIFU can reach tumours in every part of the liver as long as the lesion is completely visualised within the focal distance of the therapeutic transducer, and accessible through a safe acoustic path. Complete tumour necrosis may be achieved even when the lesion is located near bile ducts, the heart or major hepatic vessels [2]. 